\title{
Semigroups Characterized by Their Generalized Fuzzy Ideals
}

\author{
Madad Khan and Saima Anis \\ Department of Mathematics, COMSATS Institute of Information Technology, Abbottabad 22060, Pakistan \\ Correspondence should be addressed to Madad Khan; madadmath@yahoo.com
}

Received 14 January 2013; Accepted 27 February 2013

Academic Editor: Feng Feng

Copyright (C) 2013 M. Khan and S. Anis. This is an open access article distributed under the Creative Commons Attribution License, which permits unrestricted use, distribution, and reproduction in any medium, provided the original work is properly cited.

We have characterized right weakly regular semigroups by the properties of their $\left(\epsilon, \epsilon \vee q_{k}\right)$-fuzzy ideals.

\section{Introduction}

Usually the models of real world problems in almost all disciplines like in engineering, medical science, mathematics, physics, computer science, management sciences, operations research, and artificial intelligence are mostly full of complexities and consist of several types of uncertainties while dealing with them in several occasion. To overcome these difficulties of uncertainties, many theories had been developed such as rough sets theory, probability theory, fuzzy sets theory, theory of vague sets, theory of soft ideals, and the theory of intuitionistic fuzzy sets. Zadeh discovered the relationships of probability and fuzzy set theory in [1] which has appropriate approach to deal with uncertainties. Many authors have applied the fuzzy set theory to generalize the basic theories of Algebra. The concept of fuzzy sets in structure of groups was given by Rosenfeld [2]. The theory of fuzzy semigroups and fuzzy ideals in semigroups was introduced by Kuroki in $[3,4]$. The theoretical exposition of fuzzy semigroups and their application in fuzzy coding, fuzzy finite state machines, and fuzzy languages was considered by Mordeson. The concept of belongingness of a fuzzy point to a fuzzy subset by using natural equivalence on a fuzzy subset was considered by Murali [5]. By using these ideas, Bhakat and Das [6, 7] gave the concept of $(\alpha, \beta)$-fuzzy subgroups by using the "belongs to" relation $\epsilon$ and "quasi-coincident with" relation $q$ between a fuzzy point and a fuzzy subgroup and introduced the concept of an $(\epsilon, \in \vee q)$-fuzzy subgroups, where $\alpha, \beta \in\{\epsilon$, $q, \in \vee q, \in \wedge q\}$ and $\alpha \neq \in \wedge q$. In particular, $(\epsilon, \in \vee q)$ fuzzy subgroup is an important and useful generalization of Rosenfeld's fuzzy subgroup. These fuzzy subgroups are further studied in $[8,9]$. The concept of $\left(\epsilon, \in \vee q_{k}\right)$-fuzzy subgroups is a viable generalization of Rosenfeld's fuzzy subgroups. Davvaz defined $\left(\epsilon, \in \vee q_{k}\right)$-fuzzy subnearrings and ideals of a near ring in [10]. Jun and Song initiated the study of $(\alpha, \beta)$-fuzzy interior ideals of a semigroup in [11] which is the generalization of fuzzy interior ideals [12]. In [13], Kazanci and Yamak studied $\left(\epsilon, \in \vee q_{k}\right)$-fuzzy bi-ideals of a semigroup.

In this paper we have characterized right regular semigroups by the properties of their right ideal, bi-ideal, generalized bi-ideal, and interior ideal. Moreover we characterized right regular semigroups in terms of their $\left(\epsilon, \epsilon \vee q_{k}\right)$ fuzzy right ideal, $\left(\epsilon, \in \vee q_{k}\right)$-fuzzy bi-ideal, $\left(\epsilon, \in \vee q_{k}\right)$-fuzzy generalized bi-ideal, $\left(\epsilon, \in \vee q_{k}\right)$-fuzzy bi-ideal, and $\left(\epsilon, \in \vee q_{k}\right)$ fuzzy interior ideals.

Throughout this paper $S$ denotes a semigroup. A nonempty subset $A$ of $S$ is called a subsemigroup of $S$ if $A^{2} \subseteq A$. A nonempty subset $J$ of $S$ is called a left (right) ideal of $S$ if $S J \subseteq I$ (JS $\subseteq I)$. $J$ is called a two-sided ideal or simply an ideal of $S$ if it is both left and right ideal of $S$. A nonempty subset $B$ of $S$ is called a generalized bi-ideal of $S$ if $B S B \subseteq B$. A nonempty subset $B$ of $S$ is called a bi-ideal of $S$ if it is both a subsemigroup and a generalized bi-ideal of $S$. A subsemigroup $I$ of $S$ is called an interior ideal of $S$ if $S I S \subseteq I$.

An semigroup $S$ is called a right weakly regular if for every $a \in S$ there exist $x, y \in S$ such that $a=$ axay.

Definition 1. For a fuzzy set $f$ of a semigroup $S$ and $t \in(0,1]$, the crisp set $U(f ; t)=\{x \in S$ such that $f(x) \geq t\}$ is called level subset of $f$. 
Definition 2. A fuzzy subset $f$ of a semigroup $S$ of the form

$$
f(y)= \begin{cases}t \in(0,1] & \text { if } y=x, \\ 0 & \text { if } y \neq x\end{cases}
$$

is said to be a fuzzy point with support $x$ and value $t$ and is denoted by $x_{t}$.

A fuzzy point $x_{t}$ is said to belong to (resp., quasi-coincident with) a fuzzy set $f$, written as $x_{t} \in f\left(\right.$ resp., $\left.x_{t} q f\right)$, if $f(x) \geq$ $t$ (resp., $f(x)+t>1)$. If $x_{t} \in f$ or $x_{t} q f$, then we write $x_{t} \in$ $\vee q f$. The symbol $\overline{\epsilon q}$ means $\in \vee q$ does not hold. For any two fuzzy subsets $f$ and $g$ of $S, f \leq g$ means that, for all $x \in S$, $f(x) \leq g(x)$.

Generalizing the concept of $x_{t} q f$, Jun $[12,14]$ defined $x_{t} q_{k} f$, where $k \in[0,1)$, as $f(x)+t+k>1 . x_{t} \in \vee q_{k} f$ if $x_{t} \in f$ or $x_{t} q_{k} f$.

\section{2. $\left(\epsilon, \in \vee q_{k}\right)$-Fuzzy Ideals in Semigroups}

Definition 3. A fuzzy subset of $S$ is called an $\left(\epsilon, \epsilon \vee q_{k}\right)$ fuzzy subsemigroup of $S$ if for all $x, y \in S$ and $t, r \in(0,1]$ the following condition holds: $x_{t} \in f$ and $y_{r} \in f$ imply $(x y)_{\min \{t, r\}} \in \vee q_{k} f$.

Lemma 4 (see [15]). Let $f$ be a fuzzy subset of $S$. Then $f$ is an $\left(\epsilon, \in \vee q_{k}\right)$-fuzzy subsemigroup of $S$ if and only if $f(x y) \geq$ $\min \{f(x), f(y),(1-k) / 2\}$.

Definition 5. A fuzzy subset $f$ of $S$ is called an $\left(\epsilon, \in \vee q_{k}\right)$ fuzzy left (right) ideal of $S$ if for all $x, y \in S$ and $t, r \in(0,1]$ the following condition holds: $y_{r} \in f$ implies $(x y)_{t} \in \vee q_{k} f\left(x_{t} \in\right.$ $f$ implies $\left.(x y)_{t} \in \vee q_{k} f\right)$.

Lemma 6 (see [15]). Let $f$ be a fuzzy subset of S. Then $f$ is an $\left(\epsilon, \in \vee q_{k}\right)$-fuzzy left (right) ideal of $S$ if and only if $f(x y) \geq$ $\min \{f(y),(1-k) / 2\}(f(x y) \geq \min \{f(x),(1-k) / 2\})$.

Definition 7. A fuzzy subsemigroup $f$ of a semigroup $S$ is called an $\left(\epsilon, \in \vee q_{k}\right)$-fuzzy bi-ideal of $S$ if for all $x, y, z \in S$ and $t, r \in(0,1]$ the following condition holds: $x_{t} \in f$ and $z_{t} \in f$ imply $(x y z)_{t} \in \vee q_{k} f$.

Lemma 8 (see [15]). A fuzzy subset $f$ of $S$ is an $\left(\epsilon, \in \vee q_{k}\right)$ fuzzy bi-ideal of $S$ if and only if it satisfies the following conditions:

(i) $f(x y) \geq \min \{f(x), f(y),(1-k) / 2\}$ for all $x, y \in S$ and $k \in[0,1)$;

(ii) $f(x y z) \geq \min \{f(x), f(z),(1-k) / 2\}$ for all $x, y, z \in S$ and $k \in[0,1)$.

Definition 9. A fuzzy subset $f$ of a semigroup $S$ is called an $\left(\epsilon, \in \vee q_{k}\right)$-fuzzy generalized bi-ideal of $S$ if for all $x, y, z \in S$ and $t, r \in(0,1]$ the following condition holds: $x_{t} \in f$ and $z_{t} \in f$ imply $(x y z)_{t} \in \vee q_{k} f$.

Lemma 10 (see [15]). A fuzzy subset $f$ of $S$ is an $(\epsilon, \epsilon$ $\left.\vee q_{k}\right)$-fuzzy generalized bi-ideal of $S$ if and only if $f(x y z) \geq$ $\min \{f(x), f(z),(1-k) / 2\}$ for all $x, y, z \in S$ and $k \in[0,1)$.
Definition 11. A fuzzy subsemigroup $f$ of a semigroup $S$ is called an $\left(\epsilon, \in \vee q_{k}\right)$-fuzzy interior ideal of $S$ if for all $x, y, z \in$ $S$ and $t, r \in(0,1]$ the following condition holds: $y_{t} \in f$ imply $(x y z)_{t} \in \vee q_{k} f$.

Lemma 12 (see [15]). A fuzzy subset $f$ of $S$ is an $\left(\epsilon, \in \vee q_{k}\right)$ fuzzy interior ideal of $S$ if and only if it satisfies the following condition:

(i) $f(x y) \geq \min \{f(x), f(y),(1-k) / 2\}$ for all $x, y \in S$ and $k \in[0,1)$;

(ii) $f(x y z) \geq \min \{f(y),(1-k) / 2\}$ for all $x, y, z \in S$ and $k \in[0,1)$.

Example 13. Let $S=\{1,2,3$,$\} be a semigroup with binary$ operation ".," as defined in the following Cayley table:

$$
\begin{array}{l|lll}
\cdot & 1 & 2 & 3 \\
\hline 1 & 1 & 1 & 1 \\
2 & 2 & 2 & 2 \\
3 & 3 & 3 & 3
\end{array}
$$

Clearly $(S, \cdot)$ is regular semigroup and $\{1\},\{2\}$, and $\{3\}$ are left ideals of $S$. Let us define a fuzzy subset $\delta$ of $S$ as

$$
\delta(1)=0.9, \quad \delta(2)=0.6, \quad \delta(3)=0.5 \text {. }
$$

Then clearly $\delta$ is an $(\epsilon, \epsilon \vee q)$-fuzzy ideal of $S$.

Lemma 14 (see [15]). A nonempty subset $R$ of a semigroup $S$ is right (left) ideal if and only if $\left(C_{R}\right)_{k}$ is an $\left(\epsilon, \in \vee q_{k}\right)$-fuzzy right (left) ideal of $S$.

Lemma 15. A nonempty subset I of a semigroup $S$ is an interior ideal if and only if $\left(C_{I}\right)_{k}$ is an $\left(\epsilon, \in \vee q_{k}\right)$-fuzzy interior ideal of $S$.

Lemma 16. A nonempty subset $B$ of a semigroup $S$ is bi-ideal if and only if $\left(C_{B}\right)_{k}$ is an $\left(\epsilon, \in \vee q_{k}\right)$-fuzzy bi-ideal of $S$.

Lemma 17. Let $f$ and $g$ be any fuzzy subsets of semigroup $S$. Then following properties hold:
(i) $\left(f \wedge_{k} g\right)=\left(f_{k} \wedge g_{k}\right)$,
(ii) $\left(f \circ{ }_{k} g\right)=\left(f_{k} \circ g_{k}\right)$.

Proof. It is straightforward.

Lemma 18. Let $A$ and $B$ be any nonempty subsets of $a$ semigroup $S$. Then the following properties hold:

(i) $\left(C_{A} \wedge_{k} C_{B}\right)=\left(C_{A \cap B}\right)_{k}$,

(ii) $\left(C_{A^{\circ}{ }^{\circ}} C_{B}\right)=\left(C_{A B}\right)_{k}$.

Proof. It is straightforward. 


\section{Characterizations of Regular Semigroups}

Theorem 19. For a semigroup $S$, the following conditions are equivalent:

(i) $S$ is regular;

(ii) $L_{1} \cap L_{2} \cap B \subseteq B L_{1} L_{2}$ for left ideals $L_{1}, L_{2}$, and bi-ideal $B$ of a semigroup $S$.

(iii) $L[a] \cap L[a] \cap B[a] \subseteq B[a] L[a] L[a]$, for some a in $S$;

Proof. (i) $\Rightarrow$ (ii): Let $S$ be regular semigroup, then for an element $a \in S$ there exists $x \in S$ such that $a=a x a$. Let $a \in L_{1} \cap L_{2} \cap B$, where $B$ is a bi-ideal and $L_{1}$ and $L_{2}$ are left ideals of $S$. So $a \in L_{1}, a \in L_{2}$, and $a \in B$.

As $a=\operatorname{axa}=\operatorname{axaxa}=\operatorname{axaxaxa} \in(B S B) S L_{1} S L_{2} \subseteq$ $B L_{1} L_{2}$. Thus $L_{1} \cap L_{2} \cap B \subseteq B L_{1} L_{2}$.

(ii) $\Rightarrow$ (iii) is obvious.

(iii) $\Rightarrow$ (i): As $a \cup S a$ and $a \cup a^{2} \cup a S a$ are left ideal and bi-ideal of $S$ generated by $a$, respectively, thus by assumption we have

$$
\begin{aligned}
& (a \cup S a) \cap(a \cup S a) \cap\left(a^{2} \cup a S a\right) \\
& \quad \subseteq\left(a \cup a^{2} \cup a S a\right)(a \cup S a)(a \cup S a) \\
& \quad \subseteq\left(a \cup a^{2} \cup a S a\right) S(a \cup S a) \\
& \quad \subseteq\left(a \cup a^{2} \cup a S a\right)(a \cup S a) \\
& =a^{2} \cup a S a \cup a^{3} \cup a^{2} S a \cup a S a^{2} \cup a S a S a \\
& \subseteq a^{2} \cup a^{3} \cup a S a .
\end{aligned}
$$

Thus $a=a^{2}=a a=a^{2} a=a a a$ or $a=a^{3}=a a a$ or $a=a x a$, for some $x$ in $S$. Hence $S$ is regular semigroup.

Theorem 20. For a semigroup $S$, the following conditions are equivalent:

\section{(i) $S$ is regular;}

(ii) $R \cap L_{1} \cap L_{2} \subseteq R L_{1} L_{2}$ for every right ideal $R$ and bi-ideal $B$ of a semigroup $S$;

(iii) $R[a] \cap L[a] \cap L[a] \subseteq R[a] L[a] L[a]$, for some a in $S$.

Proof. (i) $\Rightarrow$ (ii): Let $S$ be regular semigroup, then for an element $a \in S$ there exists $x \in S$ such that $a=a x a$. Let $a \in R \cap L_{1} \cap L_{2}$, where $R$ is right ideal and $L_{1}$, and $L_{2}$ are left ideals of $S$. So $a \in R, a \in L_{1}$ and $a \in L_{2}$. As $a=$ axaxa $\epsilon$ (RS) $L_{1} S L_{2} \subseteq R L_{1} L_{2}$. Thus $R \cap L_{1} \cap L_{2} \subseteq R L_{1} L_{2}$.

(ii) $\Rightarrow$ (iii) is obvious. (iii) $\Rightarrow$ (i): As $a \cup a S$ is right ideal and $a \cup S a$ is left ideal of $S$ generated by $a$, respectively, thus by assumption we have

$$
\begin{aligned}
& (a \cup S a) \cap(a \cup a S) \cap(a \cup a S) \\
& \quad \subseteq(a \cup a S)(a \cup S a)(a \cup S a) \\
& \quad \subseteq(a \cup a S) S(a \cup S a) \\
& \quad \subseteq(a \cup a S)(a \cup S a) \\
& =a^{2} \cup a S a \cup a S a \cup a S S a \\
& \subseteq a^{2} \cup a S a .
\end{aligned}
$$

Thus $a=a^{2}$ or $a=a x a$, for some $x$ in $S$. Hence $S$ is regular semigroup.

Theorem 21. For a semigroup $S$, the following conditions are equivalent:

(i) $S$ is regular;

(ii) $f \circ{ }_{k} g \circ{ }_{k} h \geq f \wedge_{k} g \wedge_{k} h$ for every $\left(\epsilon, \in \vee q_{k}\right)$-fuzzy right ideal $f,\left(\in, \in \vee q_{k}\right)$-fuzzy left ideals $g$, and $h$ of $a$ semigroup $S$.

Proof. (i) $\Rightarrow$ (ii): Let $f$ be $\left(\epsilon, \in \vee q_{k}\right)$-fuzzy right ideal, $g$ and $h$ any $\left(\epsilon, \in \vee q_{k}\right)$-fuzzy left ideals of $S$. Since $S$ is regular, therefore for each $a \in S$ there exists $x \in S$ such that

$$
a=a \times a=a x a x a
$$

Thus

$$
\begin{aligned}
& \left(f \circ \circ_{k} \circ_{k} h\right)(a) \\
& =(f \circ g \circ h)(a) \wedge \frac{1-k}{2} \\
& =\left({ }_{a=p q}\{f(p) \wedge(g \circ h)(q)\}\right) \wedge \frac{1-k}{2} \\
& \geq f(a) \wedge(g \circ h)((x a)(x a)) \wedge \frac{1-k}{2} \\
& \geq f(a) \wedge((x a)(x a)=b c\{g(b) \wedge h(c)\}) \wedge \frac{1-k}{2} \\
& \geq f(a) \wedge g(x a) \wedge h(x a) \wedge \frac{1-k}{2} \\
& \geq f(a) \wedge g(a) \wedge h(a) \wedge \frac{1-k}{2} \\
& \geq f(a) \wedge g(a) \wedge h(a) \wedge \frac{1-k}{2} .
\end{aligned}
$$

(ii) $\Rightarrow$ (i): Let $R[a]$ be right ideal, and let $L_{1}[a]$ and $L_{2}[a]$ be any two left ideals of $S$ generated by $a$, respectively.

Then $\left(C_{R[a]}\right)_{k}$ is any $\left(\epsilon, \in \vee q_{k}\right)$-fuzzy right ideal, and $\left(C_{L_{1}[a]}\right)_{k}$ and $\left(C_{L_{2}[a]}\right)_{k}$ are any $\left(\epsilon, \in \vee q_{k}\right)$-fuzzy left ideals of 
semigroup $S$, respectively. Let $a \in S$ and $b \in R[a] \cap L_{1}[a] \cap$ $L_{2}[a]$. Then $b \in R[a], b \in L_{1}[a]$, and $b \in L_{2}[a]$. Now

$$
\begin{aligned}
\frac{1-k}{2} & \leq\left(C_{R[a] \cap L_{1}[a] \cap L_{2}[a]}\right)_{k}(b) \\
& =\left(\left(C_{R[a]}\right)_{k} \wedge_{k}\left(C_{L_{1}[a]}\right)_{k} \wedge_{k}\left(C_{L_{2}[a]}\right)_{k}\right)(b) \\
& \leq\left(\left(C_{R[a]}\right)_{k}{ }^{\circ}\left(C_{L_{1}[a]}\right)_{k}{ }^{\circ}\left(C_{L_{2}[a]}\right)_{k}\right)(b) \\
& =\left(C_{R[a] L_{1}[a] L_{2}[a]}\right)_{k}(b) .
\end{aligned}
$$

Thus $b \in R[a] L_{1}[a] L_{2}[a]$. Therefore $R[a] \cap L_{1}[a] \cap$ $L_{2}[a] \subseteq R[a] L_{1}[a] L_{2}[a]$.

So by Theorem $20, S$ is regular.

\section{Characterizations of Right Weakly Regular Semigroups in Terms of $\left(\epsilon, \in \vee q_{k}\right)$-Fuzzy Ideals}

Theorem 22. For a semigroup $S$, the following conditions are equivalent:

(i) $S$ is right weakly regular;

(ii) $R \cap L \cap I \subseteq R L I$ for every right ideal, left ideal, and interior ideal of S, respectively;

(iii) $R[a] \cap L[a] \cap I[a] \subseteq R[a] L[a] I[a]$.

Proof. (i) $\Rightarrow$ (ii): Let $S$ be right weakly regular semigroup, and let $R, L$, and $I$ be right ideal, left ideal, and interior ideal of $S$, respectively. Let $a \in R \cap L \cap I$ then $a \in R, a \in L$, and $a \in I$. Since $S$ is right weakly regular semigroup so for $a$ there exist $x, y \in S$ such that

$$
\begin{aligned}
a & =\operatorname{axay}=\text { axaxayy }=\text { axaxaxayy } \\
& \in(R S)(S L)(S I S) \subseteq R L I .
\end{aligned}
$$

Therefore $a \in R L I$. So $R \cap L \cap I \subseteq R L I$.

(ii) $\Rightarrow$ (iii) is obvious.

(iii) $\Rightarrow$ (i): As $a \cup a S, a \cup S a$, and $a \cup a^{2} \cup S a S$ are right ideal, left ideal, and interior ideal of $S$ generated by an element $a$ of $S$, respectively, thus by assumption, we have

$$
\begin{aligned}
(a \cup a S) & \cap(a \cup S a) \cap\left(a \cup a^{2} \cup S a S\right) \\
\subseteq & (a \cup a S)(a \cup S a)\left(a \cup a^{2} \cup S a S\right) \\
= & \left(a^{2} \cup a S a \cup a S a \cup a S S a\right)\left(a \cup a^{2} \cup S a S\right) \\
= & a^{3} \cup a^{4} \cup a^{2} S a S \cup a S a^{2} \cup a S a^{3} \cup a S a S a S \\
& \cup a S S a^{2} \cup a S S a^{3} \cup a S S a S a S \\
\subseteq & a^{3} \cup a^{4} \cup a S a \cup a S a S .
\end{aligned}
$$

Thus $a=a^{3}=a a a=a a a^{3}=a a a^{2} a$ or $a=a x a=\operatorname{axaxa}$ or $a=$ auav, for some $x, u, v$ in $S$. Hence $S$ is right weakly regular semigroup.
Theorem 23. For a semigroup $S$, the following conditions are equivalent:

(i) $S$ is right weakly regular;

(ii) $f \wedge g \wedge h \leq f \circ g \circ h$ for every fuzzy right ideal, fuzzy left ideal, and fuzzy interior ideal of $S$, respectively.

Proof. (i) $\Rightarrow$ (ii): Let $f, g$, and $h$ be any $\left(\epsilon, \in \vee q_{k}\right)$-fuzzy right ideal, $\left(\epsilon, \epsilon \vee q_{k}\right)$-fuzzy generalized bi-ideal, and $(\epsilon, \epsilon$ $\vee q_{k}$ )-fuzzy interior ideal of $S$. Since $S$ is right weakly regular therefore for each $a \in S$ there exist $x, y \in S$ such that

$$
\begin{aligned}
a & =\text { axay }=(\text { axay })(x a y)=(a x)(a y)(x a y) \\
& =(a x)(\text { axayy })(x a y)=(a x)(a x a)(\text { yyxay }) .
\end{aligned}
$$

Then

$$
\begin{aligned}
& \left(f \circ{ }_{k} g^{\circ} h\right)(a) \\
& \quad=(f \circ g \circ h)(a) \wedge \frac{1-k}{2} \\
& \quad=\left({ }_{a=p q}\{f(p) \wedge(g \circ h)(q)\}\right) \wedge \frac{1-k}{2} \\
& \geq f(a x) \wedge(g \circ h)((a x a)(\text { yyxay })) \wedge \frac{1-k}{2} \\
& \geq f(a) \wedge((a x a)(\text { yyxay })=b c\{g(b) \wedge h(c)\}) \wedge \frac{1-k}{2} \\
& \geq f(a) \wedge g(a x a) \wedge h(y y x a y) \wedge \frac{1-k}{2} \\
& \geq f(a) \wedge g(a) \wedge h(a) \wedge \frac{1-k}{2} \\
& \geq f(a) \wedge g(a) \wedge h(a) \wedge \frac{1-k}{2} .
\end{aligned}
$$

Therefore $f \wedge_{k} g \wedge_{k} h \leq f{ }^{\circ}{ }_{k}{ }^{\circ}{ }_{k} h$.

Now (ii) $\Rightarrow$ (i)

(ii) $\Rightarrow$ (i): Let $R[a], L[a]$, and $I[a]$ be right ideal, left ideal, and interior ideal of $S$ generated by $a$, respectively.

Then $\left(C_{R[a]}\right)_{k},\left(C_{L[a]}\right)_{k}$, and $\left(C_{I[a]}\right)_{k}$ are $\left(\epsilon, \in \vee q_{k}\right)$-fuzzy right ideal, $\left(\epsilon, \in \vee q_{k}\right)$-fuzzy left ideal, and $\left(\epsilon, \in \vee q_{k}\right)$-fuzzy interior ideal of semigroup $S$. Let $a \in S$ and $b \in R[a] \cap L[a] \cap$ $I[a]$. Then $b \in R[a], b \in L[a]$, and $b \in I[a]$. Now

$$
\begin{aligned}
\frac{1-k}{2} & \leq\left(C_{R[a] \cap L[a] \cap[a]}\right)_{k}(b) \\
& =\left(\left(C_{R[a]}\right)_{k} \wedge_{k}\left(C_{L[a]}\right)_{k} \wedge_{k}\left(C_{I[a]}\right)_{k}\right)(b) \\
& \leq\left(\left(C_{R[a]}\right)_{k} \circ_{k}\left(C_{L[a]}\right)_{k} \circ_{k}\left(C_{I[a]}\right)_{k}\right)(b) \\
& =\left(C_{R[a] L[a][[a]}\right)_{k}(b) .
\end{aligned}
$$

Thus $b \in R[a] L[a] I[a]$. Therefore $R[a] \cap L[a] \cap I[a] \subseteq$ $R[a] L[a] I[a]$. Hence by Theorem $22, S$ is right weakly regular semigroup. 
Theorem 24. For a semigroup $S$, the following conditions are equivalent:

(i) $S$ is right weakly regular;

(ii) $B \cap L \cap I \subseteq B L I$ for every bi-ideal, left ideal, and interior ideal of $S$, respectively;

(iii) $B[a] \cap L[a] \cap I[a] \subseteq B[a] L[a] I[a]$.

Proof. (i) $\Rightarrow$ (ii): Let $S$ be right weakly regular semigroup, and $B, L$, and $I$ be bi-ideal, left ideal, and interior ideal of $S$, respectively. Let $a \in B \cap L \cap I$ then $a \in B, a \in L$, and $a \in I$. Since $S$ is right weakly regular semigroup so for $a$ there exist $x, y \in S$ such that

$$
\begin{aligned}
a & =\text { axay }=\text { axaxaxayy } \\
& =(\text { axa })(x a)(\text { xayyy }) \in(B S)(S L)(S I S) \subseteq B L I .
\end{aligned}
$$

Therefore $a \in B L I$.So $B \cap L \cap I \subseteq B L I$.

(ii) $\Rightarrow$ (iii) is obvious.

(iii) $\Rightarrow$ (i): As $a \cup a^{2} \cup a S a, a \cup S a$, and $a \cup a^{2} \cup S a S$ are bi-ideal, left ideal, and interior ideal of $S$ generated by an element $a$ of $S$, respectively, thus by assumption we have

$$
\begin{aligned}
(a \cup & \left.a^{2} \cup a S a\right) \cap(a \cup S a) \cap\left(a \cup a^{2} \cup S a S\right) \\
\subseteq & \left(a \cup a^{2} \cup a S a\right)(a \cup S a)\left(a \cup a^{2} \cup S a S\right) \\
= & \left(a^{2} \cup a S a \cup a^{3} \cup a^{2} S a \cup a S a^{2} \cup a S a S a\right) \\
& \times\left(a \cup a^{2} \cup S a S\right) \\
= & a^{3} \cup a^{4} \cup a^{2} S a S \cup a S a^{2} \cup a S a^{3} \cup a S a S a S \cup a^{4} \cup a^{5} \\
& \cup a^{3} S a S \cup a^{2} S a^{2} \cup a^{2} S a^{3} \cup a^{2} S a S a S \cup a S a^{3} \\
& \cup a S a^{4} \cup a S a^{2} S a S a S a S a^{2} \\
& \cup a S a S a^{3} \cup a S a S a S a S \\
\subseteq & a^{3} \cup a^{4} \cup a^{5} \cup a S a \cup a S a S .
\end{aligned}
$$

Thus $a=a^{4}=a a a a$ or $a=a^{3}=a a a=a a a^{3}=a a a^{2} a$ or $a=a x a=\operatorname{axaxa}$ or $a=a u a v$, for some $x, u, v$ in $S$. Hence $S$ is right weakly regular semigroup.

Theorem 25. For a semigroup $S$, the following conditions are equivalent:

(i) $S$ is right weakly regular;

(ii) $f \wedge g \wedge h \leq f \circ g \circ h$ for every fuzzy bi-ideal, fuzzy left ideal and fuzzy interior ideal of $S$, respectively;

(iii) $f \wedge g \wedge h \leq f \circ g \circ h$ for every fuzzy generalized bi-ideal, fuzzy left ideal, and fuzzy interior ideal of $S$, respectively.
Proof. (i) $\Rightarrow$ (iii): Let $f, g$, and $h$ be any $\left(\epsilon, \in \vee q_{k}\right)$-fuzzy generalized bi-ideal, $\left(\epsilon, \epsilon \vee q_{k}\right)$-fuzzy left ideal, and $(\epsilon, \epsilon$ $\vee q_{k}$ )-fuzzy interior ideal of $S$. Since $S$ is right weakly regular for each $a \in S$ there exist $x, y \in S$ such that

$$
\begin{aligned}
a & =a x a y=(a x a y)(x a y)=(a x)(a y)(x a y) \\
& =(a x)(a x a y y)(x a y)=(a x a)(x a)(y y x a y) .
\end{aligned}
$$

Then

$$
\begin{aligned}
& \left(f \circ{ }_{k} g \circ h\right)(a) \\
& \quad=(f \circ g \circ h)(a) \wedge \frac{1-k}{2} \\
& =\left(_{a=p q}\{f(p) \wedge(g \circ h)(q)\}\right) \wedge \frac{1-k}{2} \\
& \geq f(a x a) \wedge(g \circ h)((x a)(y y x a y)) \wedge \frac{1-k}{2} \\
& \geq f(a) \wedge((x a)(y y x a y)=b c\{g(b) \wedge h(c)\}) \wedge \frac{1-k}{2} \\
& \geq f(a) \wedge g(x a) \wedge h(y y x a y) \wedge \frac{1-k}{2} \\
& \geq f(a) \wedge g(a) \wedge h(a) \wedge \frac{1-k}{2} \\
& \geq f(a) \wedge g(a) \wedge h(a) \wedge \frac{1-k}{2} .
\end{aligned}
$$

Therefore $f \wedge_{k} g \wedge_{k} h \leq f{ }_{k} g \circ{ }_{k} h$.

(iii) $\Rightarrow$ (ii) is obvious.

(ii) $\Rightarrow$ (i): Let $B[a], L[a]$, and $I[a]$ be bi-ideal, left ideal, and interior ideal of $S$ generated by $a$, respectively.

Then $\left(C_{B[a]}\right)_{k},\left(C_{L[a]}\right)_{k}$, and $\left(C_{I[a]}\right)_{k}$ are $\left(\epsilon, \in \vee q_{k}\right)$-fuzzy bi-ideal, $\left(\epsilon, \in \vee q_{k}\right)$-fuzzy left ideal, and $\left(\epsilon, \in \vee q_{k}\right)$-fuzzy interior ideal of semigroup $S$. Let $a \in S$ and $b \in B[a] \cap L[a] \cap$ $I[a]$. Then $b \in B[a], b \in L[a]$, and $b \in I[a]$. Now

$$
\begin{aligned}
\frac{1-k}{2} & \leq\left(C_{B[a] \cap L[a] \cap[[a]}\right)_{k}(b) \\
& =\left(\left(C_{B[a]}\right)_{k} \wedge_{k}\left(C_{L[a]}\right)_{k} \wedge_{k}\left(C_{I[a]}\right)_{k}\right)(b) \\
& \leq\left(\left(C_{B[a]}\right)_{k} \circ_{k}\left(C_{L[a]}\right)_{k} \circ_{k}\left(C_{I[a]}\right)_{k}\right)(b) \\
& =\left(C_{B[a] L[a] I[a]}\right)_{k}(b) .
\end{aligned}
$$

Thus $b \in B[a] L[a] I[a]$. Therefore $B[a] \cap L[a] \cap I[a] \subseteq$ $B[a] L[a] I[a]$. Hence by Theorem $24, S$ is right weakly regular semigroup.

Theorem 26. For a semigroup $S$, the following conditions are equivalent:

(i) $S$ is right weakly regular;

(ii) $Q \cap L \cap I \subseteq Q L I$ for every quasi-ideal $Q$, left ideal $L$, and interior ideal I of S, respectively;

(iii) $Q[a] \cap L[a] \cap I[a] \subseteq Q[a] L[a] I[a]$. 
Proof. (i) $\Rightarrow$ (ii): Let $S$ be right weakly regular semigroup, and let $Q, L$, and $I$ be quasi-ideal, left ideal, and interior ideal of $S$, respectively. Let $a \in Q \cap L \cap I$ then $a \in Q, a \in L$, and $a \in I$. Since $S$ is right weakly regular semigroup so for $a$ there exist $x, y \in S$ such that

$$
\begin{aligned}
a & =a x a y=(\text { axay })(x a y) \\
& =a(x a)(y x a y) \in Q(S L)(S I S) \subseteq Q L I .
\end{aligned}
$$

Therefore $a \in Q L I$. So $Q \cap L \cap I \subseteq Q L I$.

(ii) $\Rightarrow$ (iii) is obvious.

(iii) $\Rightarrow($ i $)$ : As $a \cup(a S \cap S a), a \cup S a$, and $a \cup a^{2} \cup S a S$ are quasi-ideal, left ideal, and interior ideal of $S$ generated by an element $a$ of $S$, respectively, thus by assumption we have

$$
\begin{aligned}
(a \cup(a S \cap S a)) \cap(a \cup S a) \cap\left(a \cup a^{2} \cup S a S\right) \\
\subseteq(a \cup(a S \cap S a))(a \cup S a)\left(a \cup a^{2} \cup S a S\right) \\
\subseteq(a \cup a S)(a \cup S a)\left(a \cup a^{2} \cup S a S\right) \\
=\left(a^{2} \cup a S a \cup a S a \cup a S S a\right)\left(a \cup a^{2} \cup S a S\right) \\
=a^{3} \cup a^{4} \cup a^{2} S a S \cup a S a^{2} \cup a S a^{3} \cup a S a S a S \\
\quad \cup a S S a^{2} \cup a S S a^{3} \cup a S S a S a S \\
\subseteq a^{3} \cup a^{4} \cup a S a \cup a S a S .
\end{aligned}
$$

Thus $a=a^{4}=a a a a$ or $a=a^{3}=a a a=a a a^{3}=a a a^{2} a$ or $a=a \times a=a x a x a$ or $a=a u a v$, for some $x, u, v$ in $S$. Hence $S$ is right weakly regular semigroup.

Theorem 27. For a semigroup $S$, the following conditions are equivalent:

(i) $S$ is right weakly regular;

(ii) $f \wedge g \wedge h \leq f \circ g \circ h$ for every fuzzy quasi-ideal, fuzzy left ideal, and fuzzy interior ideal of $S$, respectively.

Proof. (i) $\Rightarrow$ (iii): Let $f, g$, and $h$ be any $\left(\epsilon, \in \vee q_{k}\right)$-fuzzy quasi-ideal, $\left(\epsilon, \in \vee q_{k}\right)$-fuzzy left ideal, and $\left(\epsilon, \in \vee q_{k}\right)$-fuzzy interior ideal of $S$. Since $S$ is right weakly regular therefore for each $a \in S$ there exist $x, y \in S$ such that

$$
a=a x a y=(a x a y)(x a y)=a(x a)(y x a y) .
$$

Then

$$
\begin{aligned}
& \left(f \circ{ }_{k} g \circ{ }_{k} h\right)(a) \\
& \quad=(f \circ g \circ h)(a) \wedge \frac{1-k}{2} \\
& \quad=\left({ }_{a=p q}\{f(p) \wedge(g \circ h)(q)\}\right) \wedge \frac{1-k}{2} \\
& \quad \geq f(a) \wedge(g \circ h)((x a)(y x a y)) \wedge \frac{1-k}{2}
\end{aligned}
$$

$$
\begin{aligned}
& \geq f(a) \wedge\left(_{(x a)(y x a y)=b c}\{g(b) \wedge h(c)\}\right) \wedge \frac{1-k}{2} \\
& \geq f(a) \wedge g(x a) \wedge h(y x a y) \wedge \frac{1-k}{2} \\
& \geq f(a) \wedge g(a) \wedge h(a) \wedge \frac{1-k}{2} \\
& \geq f(a) \wedge g(a) \wedge h(a) \wedge \frac{1-k}{2} .
\end{aligned}
$$

Therefore $f \wedge_{k} g \wedge_{k} h \leq f{ }_{k} g \circ{ }_{k} h$.

(iii) $\Rightarrow$ (ii) is obvious.

(ii) $\Rightarrow$ (i): Let $Q[a], L[a]$, and $I[a]$ be quasi-ideal, left ideal and interior ideal of $S$ generated by $a$, respectively.

Then $\left(C_{\mathrm{Q}[a]}\right)_{k},\left(C_{L[a]}\right)_{k}$, and $\left(C_{I[a]}\right)_{k}$ are $\left(\epsilon, \in \vee q_{k}\right)$-fuzzy quasi-ideal, $\left(\epsilon, \in \vee q_{k}\right)$-fuzzy left ideal, and $\left(\epsilon, \in \vee q_{k}\right)$-fuzzy interior ideal of semigroup $S$. Let $a \in S$ and let $b \in Q[a] \cap$ $L[a] \cap I[a]$. Then $b \in Q[a], b \in L[a]$, and $b \in I[a]$. Now

$$
\begin{aligned}
\frac{1-k}{2} & \leq\left(C_{\mathrm{Q}[a] \cap L[a] \cap I[a]}\right)_{k}(b) \\
& =\left(\left(C_{\mathrm{Q}[a]}\right)_{k} \wedge_{k}\left(C_{L[a]}\right)_{k} \wedge_{k}\left(C_{I[a]}\right)_{k}\right)(b) \\
& \leq\left(\left(C_{\mathrm{Q}[a]}\right)_{k} \circ_{k}\left(C_{L[a]}\right)_{k} \circ_{k}\left(C_{I[a]}\right)_{k}\right)(b) \\
& =\left(C_{\mathrm{Q}[a] L[a] I[a]}\right)_{k}(b) .
\end{aligned}
$$

Thus $b \in Q[a] L[a] I[a]$. Therefore $Q[a] \cap L[a] \cap I[a] \subseteq$ $Q[a] L[a] I[a]$. Hence by Theorem $26, S$ is right weakly regular semigroup.

\section{References}

[1] L. A. Zadeh, "Fuzzy sets," Information and Computation, vol. 8, pp. 338-353, 1965.

[2] A. Rosenfeld, "Fuzzy groups," Journal of Mathematical Analysis and Applications, vol. 35, pp. 512-517, 1971.

[3] N. Kuroki, "Fuzzy bi-ideals in semigroups," Commentarii Mathematici Universitatis Sancti Pauli, vol. 28, no. 1, pp. 17-21, 1980.

[4] N. Kuroki, "On fuzzy ideals and fuzzy bi-ideals in semigroups," Fuzzy Sets and Systems, vol. 5, no. 2, pp. 203-215, 1981.

[5] V. Murali, "Fuzzy points of equivalent fuzzy subsets," Information Sciences, vol. 158, pp. 277-288, 2004.

[6] S. K. Bhakat and P. Das, "On the definition of a fuzzy subgroup," Fuzzy Sets and Systems, vol. 51, no. 2, pp. 235-241, 1992.

[7] S. K. Bhakat and P. Das, “( $\epsilon, \epsilon, V q)$-fuzzy subgroup," Fuzzy Sets and Systems, vol. 80, no. 3, pp. 359-368, 1996.

[8] S. K. Bhakat and P. Das, "Fuzzy subrings and ideals redefined," Fuzzy Sets and Systems, vol. 81, no. 3, pp. 383-393, 1996.

[9] S. K. Bhakat, “ $(\epsilon, V q)$-level subset," Fuzzy Sets and Systems, vol. 103, no. 3, pp. 529-533, 1999.

[10] B. Davvaz, “ $(\epsilon, \epsilon, V q)$-fuzzy subnear-rings and ideals," Soft Computing, vol. 10, no. 3, pp. 206-211, 2006.

[11] Y. B. Jun and S. Z. Song, "Generalized fuzzy interior ideals in semigroups," Information Sciences, vol. 176, no. 20, pp. 30793093, 2006.

[12] Y. B. Jun, "New types of fuzzy subgroups," submitted. 
[13] O. Kazanci and S. Yamak, "Generalized fuzzy bi-ideals of semigroup," Soft Computing, vol. 12, pp. 1119-1124, 2008.

[14] Y. B. Jun, "Generalizations of $(\epsilon, \epsilon, V q)$-fuzzy subalgebras in BCK/BCI-algebras," Computers \& Mathematics with Applications, vol. 58, no. 7, pp. 1383-1390, 2009.

[15] M. Shabir, Y. B. Jun, and Y. Nawaz, "Semigroups characterized by $(\epsilon, \epsilon, V q k)$-fuzzy ideals," Computers \& Mathematics with Applications, vol. 60, no. 5, pp. 1473-1493, 2010. 


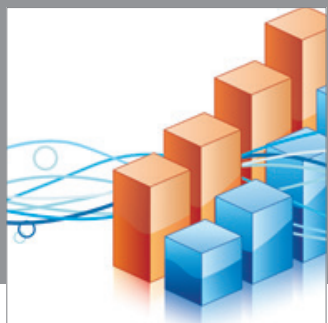

Advances in

Operations Research

mansans

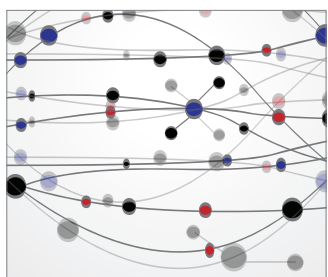

The Scientific World Journal
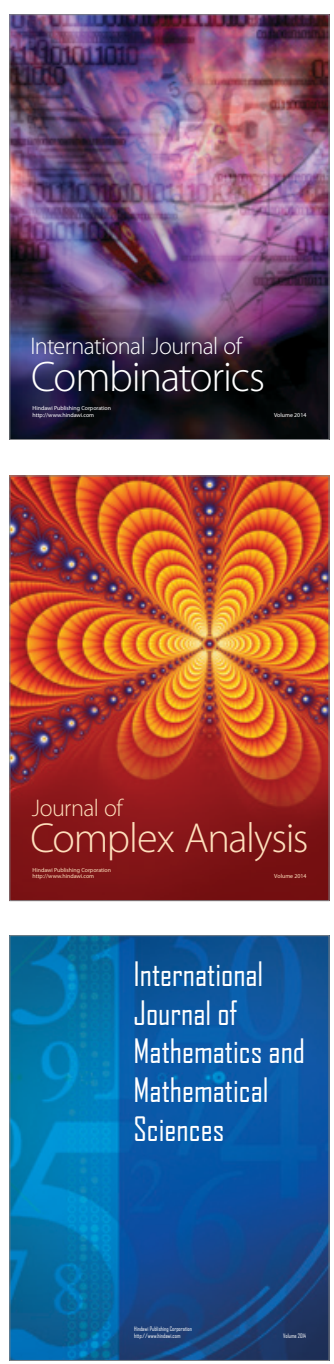
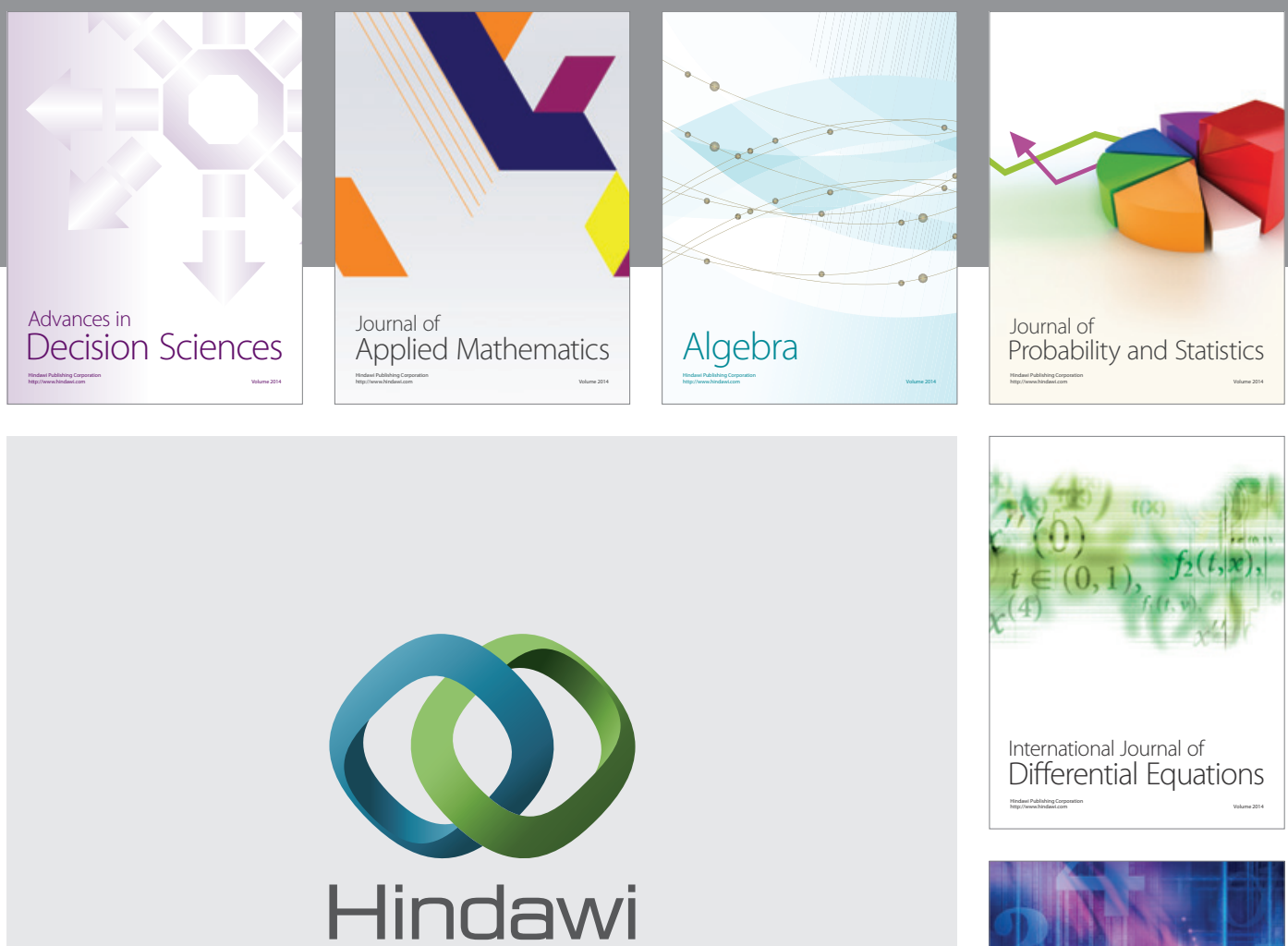

Submit your manuscripts at http://www.hindawi.com
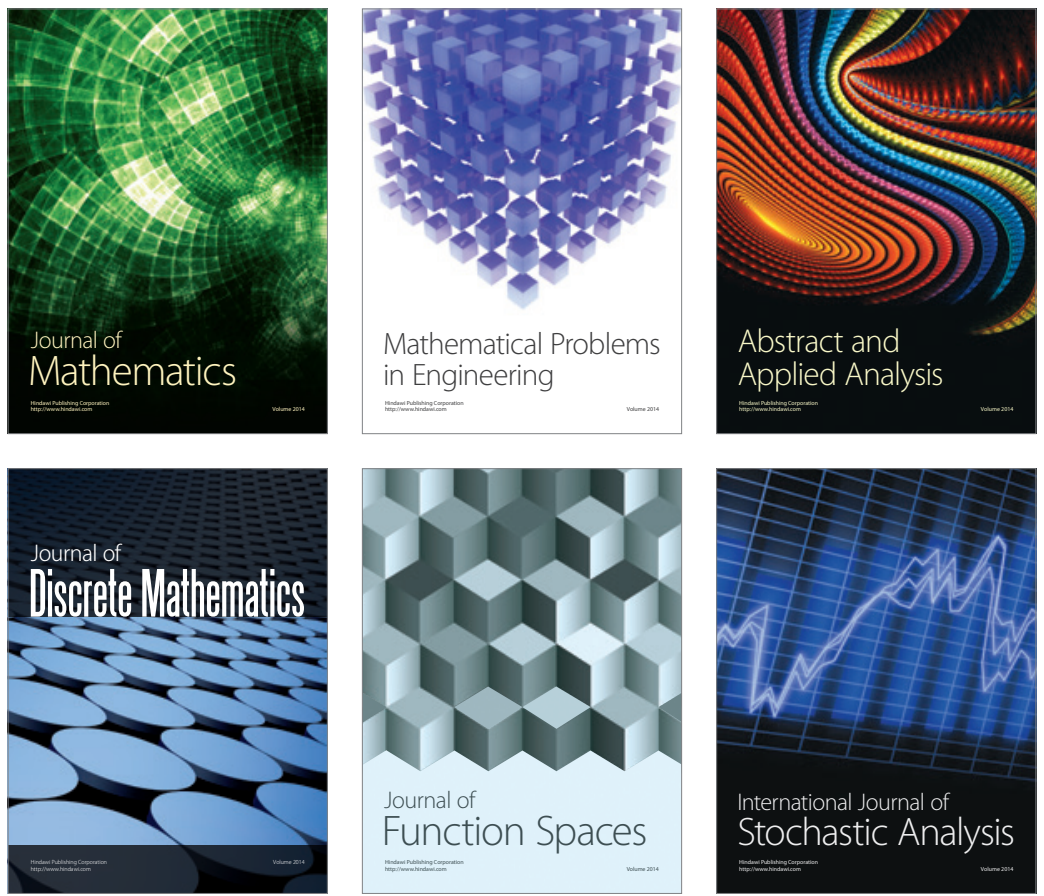

Journal of

Function Spaces

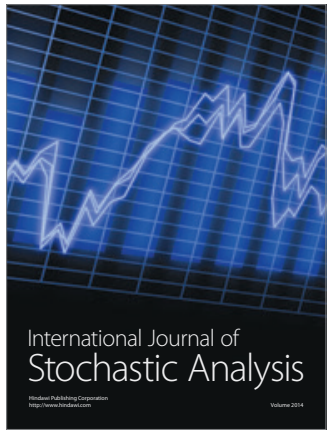

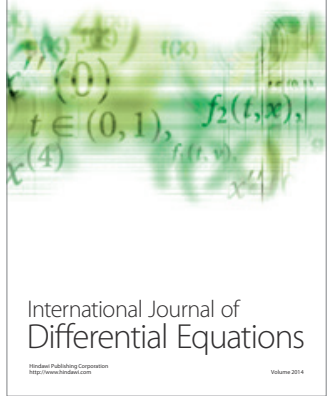
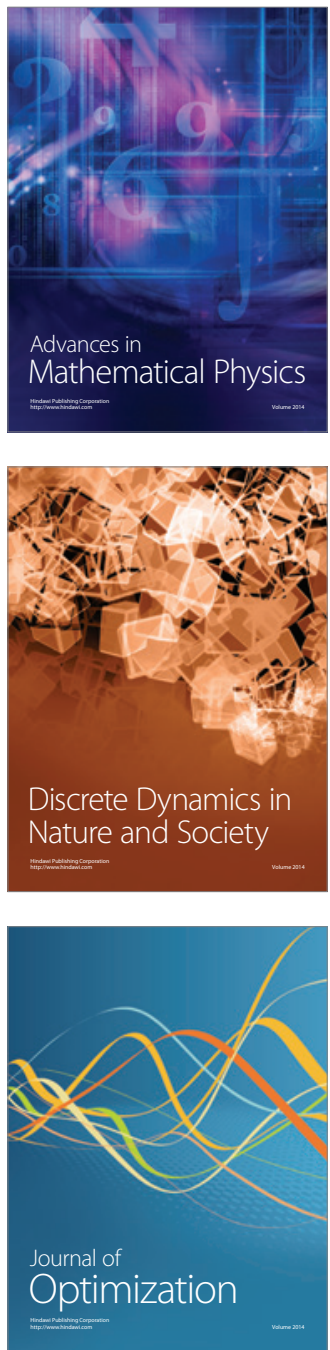